\title{
Treat Me Well: Affective and Physiological Feedback for Wheelchair Users
}

\author{
Octavian Postolache \\ Instituto de \\ Telecomunicações \\ ISCTE-IUL, \\ Lisbon, Portugal \\ opostolache@1x.it.pt
}

\author{
Pedro Silva Girão, \\ Mário Ribeiro \\ Instituto de \\ Telecomunicações \\ Lisbon, Portugal \\ psgirao@ist.utl.pt
}

\author{
Helder Carvalho, \\ André Catarino, \\ Universidade do Minho, \\ Braga, Portugal \\ helder@det.uminho.pt
}

\author{
Gabriela Postolache \\ Universidade Atlântica, \\ Oeiras, Portugal \\ gabrielap@uatlantica.pt
}

\begin{abstract}
This work reports a electrocardiograph and skin conductivity hardware architecture, based on E-textile electrodes, attached to a wheelchair for affective and physiological computing. Appropriate conditioning circuits and a microcontroller platform that performs acquisition, primary processing, and communication using Bluetooth were designed and implemented. To increase the accuracy and repeatability of the skin conductivity measuring channel, force measurement sensors were attached to the system certifying measuring contact force on the electrode level. Advanced processing including Rwave peak detector, adaptive filtering and autonomic nervous system analysis based on wavelets transform was designed and implemented on a server. A central design of affective recognition and biofeedback system is described.
\end{abstract}

Keywords - affective computing, physiological computing, embedded system, E-textile, heart rate variability

\section{INTRODUCTION}

Mrs. Alda (fictitious name) is an old age woman with elegant body posture and clothes. Age traces in her clear pink skin are faded by brilliance of her blue tiny eyes remembering the many grannies who with goodness and wisdom reflected in the hands and body movements, gathered around the warm cakes and fascinating stories, the grandchildren, the family, the community. She is an 88 years old lady invited to a Nursing Workshop to speak about her experience on retirement residence where she has been living for the past 5 years. She says that she feels well mainly when she can receive her family visits and when she can talk with the nurse. When asked to indicate what she desires to demand from any nurse from a retirement residence, she says shortly, with a smile that seems guilty, that what she wants is to be "well treated". Nobody ask her to give examples of what she means by "well treated", perhaps because before her presence on the workshop, was analyzed a text from a letter of a American nurse, Mrs. Rosemary Wills, who in order to prepare her future in a nursing home, wrote on the needs of aged people and on the attitude that nurses should have in order to respect old people's dignity. It is outlined in this letter that - "Perhaps it will seem demanding, exaggerated in what I want, but what I want is just to receive affection, to be well treated, and a friendly, lovely person to take charge of me".

Our team has been involved in the development of electronic health systems that allow non-invasive or unobtrusive health and healthcare monitoring. Currently, we investigate developed or in course of implementation technologies that should allow affective communication, a perception of well treatment, and might turn the caregivers more friendly and lovely.

In our work we search for solution to a range of issues related with affective and physiological computing for wheelchair users: What emotions or moods should be outlined, when, and how? Are deep models of emotion necessary? The programed software should respond to all situations or can respond only to some events and selected requirements? How the affective expressions maintain subject engagement? Which expressive modalities should be used (e.g. face expressions, gestures, behavior selection)? What role do the subject's emotions play in the developed affective and physiological sensing? Does the personality of the involved people must be assessed in affective computing? What information about the person affective reactions should enable adaptations to the daily life challenges?

The aim of this work is to explore the rich possibilities of affective computing and physiological computing in order to set out new research for future healthcare solutions. In Sections 2 and 3 of the paper a general introduction on affective and physiological computing is presented. In Section 4 we introduce our work on unobtrusive psychophysiological interactive computer system implementation. Finally, we conclude with future directions of research.

\section{AFFECTIVE COMPUTING}

Affective Computing is a "branch of study and development of artificial intelligence that deals with the design of systems and devices that can recognize, interpret, and process human emotions" [1]. The pioneer of this type of research, Rosalind Picard, defines Affective Computing as "computing that relates to, arises from, or deliberately influences emotion" [2]. 
Emotions are seen by many scientists as a particular kind of subjective feeling (the affective aspect of consciousness), as a complex psychophysiological experience of an individual's state of mind as interacting with biochemical and environmental influences, or bio-regulatory reactions aimed at the promotion, directly or indirectly, of the sort of physiological states associated with feeling which is manifested in complex interactions between individuals and their environments over time. These psychophysiological patterns are both affected by and have effect across multiple levels of analysis, including genotypic, endophenotypic, phenotypic, behavioral, and social. Given this, no single theory can be expected to account for any particular emotion in general. Antonio Damasio, who contributed to demonstrate that emotions play a critical role in high level cognition, makes an important distinction between primary or early emotions, which are innate or "hard-wired" (and are thus essentially universal) and secondary or adult emotions, which derive from experience and acquired knowledge (and are thus highly individualized.) We born with "pre-programmed" emotions - the primary emotions - as pleasure of being hold in warm arms or sorrow to a tissue lesion - but we learn the much more complex responses - the secondary emotions - based on our experiences, particularly in childhood and adolescence, although the process of developing secondary emotions continues throughout our entire lives. We learn these emotions as we associate certain stimuli with positive or negative outcomes, although it is obvious that the process of making these associations is by no means full proof. Emotions can be categorized as pleasant/appetitive or approach versus unpleasant/aversive or avoidance. Based on extensive animal and pharmacological work, Gray proposed a behavioral approach system (BAS) and a behavioral inhibition system (BIS) [3]. The BAS governs appetitive behaviors in response to reward, resulting in post-event (stimuli) pleasurable affective state. In contrast to the BAS, the BIS inhibits prepotent behaviors when conflict arises due to competing motivational objectives. These competing objectives may represent approach-approach conflicts, avoidance-avoidance conflicts, or approach-avoidance conflicts. BIS activation induces anxiety, which facilitates behaviors aimed at resolving the divergent motivational goals. Excessive BIS activity has been linked to heightened risk for internalizing disorders, whereas deficient BIS activity has been linked to behavioral disinhibition [3]. Moreover, "any stimulus that has the potential for increasing an organism's experience of safety, has the potential of recruiting the evolutionarily more advanced neural circuits that support the pro-social behaviors of the social engagement system" [4], for social interaction and communication - like empathy or "feeling of others and to be sensitive to other's different beliefs and perspective". Often characterized as the ability to "put oneself into another's shoes" empathizing is a socio-emotional response defined as a capacity to attribute mental state, such as emotions, thoughts, and intentions to other people, and to respond to their mental states with an appropriate emotion. Therefore, the affective computing should allow perception, interpretation of human feeling, exploiting a more natural set of communication channels and hence to facilitate intelligent and sensitive response, and the adaptation of the task or the interaction to the state of users. The multi-component character of emotions and its consideration in the area of human-technology interaction was discussed by Mahlke et al. [5]. They investigated five components of emotional experience in an interactive context, namely: subjective feelings, physiological activation, motor expression, cognitive appraisals, and behavioral tendencies. However, a more comprehensive and functional model of a system for real-time multimodal analysis of nonverbal affective social interaction in usercentric media was presented by the Varni team [6]. The system is composed by 1) the Analysis Component that performs data acquisition, preprocessing, and extraction of low level and high level affective features from each user in a synchronized way, 2) the Social Interaction Analysis Component that computes the level of synchronization of affective behavior and leadership among the users, starting from the affective characterizations the Analysis Component provides, 3) the Multimedia Content Processing Component that applies the processing techniques for shaping and molding multimedia content in real time.

Measuring affective features is challenging. Despite many advances in brain imaging, there is not yet any low cost, unobtrusive technology that can read feeling and thought and communicate appropriately it. There is a long history of feeling measurement in psychology, being the first evidence documented as far as in the period of 290 to 280 B.C. It was Eristratus, the grandson of Aristotle, who diagnosed the love of Anthiochus for his mother by operationalizing emotion with the following dependent variables: voice quality, eye movements, skin responses, and blood pressure. Nowadays, typical instruments used for emotion measurement in psychophysiology are questionnaires, adjectives, images, role playing, as well as a set of tools that measure different physiological responses. Affecting Computing expands these options, enabling new kinds of automatic, mobile sometimes real-time measurements using features of voice, language, facial expression, posture, gesture, heart rate, temperature, respiration, heart rate variability, electrodermal response, blood pressure, etc. The more components are to be measured, the more data is available to interpret the user's reactions.

Many systems for affective computing use wearable sensors that can capture some affective features (e.g. facial expression, vocal inflections changes, heart rate, electrodermal activity, gesture, posture, etc.). Wearable systems are becoming smaller, but there are still issues with the number of wires flowing in and out of the system and sensors slipping of. There are only a few prototypes that unobtrusively might measure affective changes. For instance, pressure sensitive mouse was implemented that can give information on frustration, caused by poor usability in a computer interface [7]; a regular office chair embedded with electromechanical film (the EMFi chair) has been shown to give information on users emotions by measuring heart rate responses [8-9]; IBM Blue-Eyes camera to detect facial expressions, Body Pressure 
Measurement System and AutoTutor's log files for conversational cues were used for integrating affect sensors in an intelligent tutoring system [8].

There are no sensors systems that can reliably, unobtrusively and continuously measure the affective state [8]. Without reliable sensors, how can we quantify exactly normal emotional changes? There is a need to develop sensors, interfaces, signal processing, pattern recognition, and reasoning algorithms for continuous tracking of person's affective interactions. These technologies will be key in assessing individual's specific areas of strength and weakness, as well as measuring the efficacy of various interventions [8]. Use of affective computing in medical practice is only in its infant phase depending on the development of more reliable wearable and unobtrusive affective sensing and computing and on the creation of synergies in the greater medical informatics/e-health research community.

\section{PHYSIOLOGICAL COMPUTING}

Physiological computing involves the direct interfacing of human physiology and computer technology [10]. Currently, physiological parameters are used as metrics and as input source for affective/emotional, health state and daily activities in human computer interfaces. The applications of physiological computing have been divided into two categories: biofeedback-based systems and bio-cybernatically adaptive systems [10]. In biofeedback applications, the role of computer is to retrieve physiological signals from the sensing hardware, pre-process the signals and display measured features of aquired physiological signals back to the subjects in real time. For instance, biofeedback based on the electromyographic signal is a method for training patients to regain muscle control lost due to accident or illness [11]. As any single aspect of detectable human physiology may by itself unreliable for affective state estimation [12-13], multichannel and multisignal devices should allow the access to more reliable indicators of physiological parameters.

Bio-cybernetic adaptation refers to the modification of system's functionality or appearance based on the real-time measurement of psychophysiology. Rather than feeding psychophysiological data back to a subject, a biocybernetically adaptive system uses the changing in psychophysiological state of the user in order to change its own functionality and/or appearance [10]. For example, a biocybernetic system may detect user's fatigue by measuring long duration eye blinks, or changing in font size and screen contrast in order to diminish visual fatigue [10]. The design logic behind bio-cybernetic adaptation may be exported to future generations of therapeutic interventions based on biofeedback in order to challenge, engage and stimulate the user.

Both affective computing and physiological computing will also require environmental and task related data in order to increase reliability and usability of these technology.

\section{SYSTEM DESCRIPTION - HARDWARE}

The goal of the present work was to develop an unobtrusive affective and physiological sensing and computing system dedicated mainly for wheelchair users low-level and highlevel affective features extraction. The implemented system performs electrocardiogram (ECG), skin conductivity (SKC), applied force on wheelchair hand support, and wheelchair acceleration acquisition. E-textile dry electrodes attached to the wheelchair were used for ECG and SKC acquisition. The acquisition of the biomedical signals, primary processing, and Bluetooth data communication are done using a designed and implemented embedded system based on a PIC24F microcontroller. The acquired data associated with wheelchair user cardiac and skin conductivity measurement is transmitted to a server application that includes an affective analysis component that generates information on emotions and the estimated psychophysiological status. Additionally, a human machine interface implemented into a tablet computer attached to the wheelchair is used for biofeedback and biocybernetically adaptive approach.

The wheelchair with cardiac and skin conductivity sensing capabilities includes a set of integrated E-textile electrodes, force sensors (FS) and a microcontroller platform with Bluetooth communication capability (Fig.1). The acquired and primary processed data is uploaded on the server that provides web pages on psychophysiological assessment that can be accessed by the wheelchair user or by care givers.

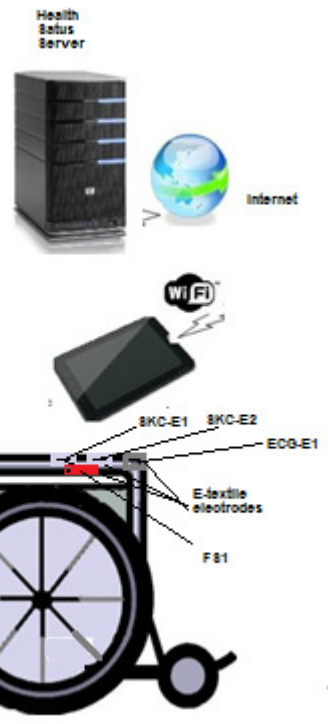

Figure 1. Smart wheelchair and healt status server architecture

Three E-textile electrodes were used for each hand support, one of them as part of a 2 lead ECG measurement channel and the other two electrodes were used to acquire the skin conductivity values. A signal conditioning circuits (CC) and microcontroller platform (acquisition, processing and communication module) were embedded on the wheelchair.

The microcontroller platform includes a 3D accelerometer used to monitor the wheelchair user activity. Acceleration 
information is used together with the force information to limit the number of false alarms caused by wheelchair motion artifacts or variation in force applied on the electrodes' surface during the skin conductivity measurement.

\section{A. E-textile electrodes}

In the last years, the quest for ubiquitous health monitoring has produced a growing interest for integration of sensors and systems into textiles. Textiles excel as interfaces for biological signal sensing as they are flexible, conformable, and users are accustomed to wearing them on a daily basis. The development of conductive yarns able to be integrated into textiles by conventional textile production processes has opened new possibilities for the development of new applications. Sensors for monitoring breathing rate and movement have been tested with knitted textiles based on conductive yarns, as described in [14-16]. Electrodes for physiological signal sensing, such as ECG, EMG (electromyography) or skin impedance have been investigated and demonstrated [16-20]. Moisture sensing for monitoring of bed-rest and disabled people has been studied [21]. In these studies some limitations of textiles as extension or moisture sensors have been observed [15][21], but generally the performance of the textile electrodes as bio-signal interfaces has been found satisfactory [16][18][20].

In this work, several types of textile electrodes have been evaluated to find the appropriate solution for ECG and SKC measuring channels. The electrodes can be differentiated by two main factors: i) the type of conductive yarn used, ii) the textile structure used in the substrate and the electrode.

Two types of conductive yarns were used in this work. The first, Bekitex 50/2, is a two-ply staple yarn based on $80 \%$ polyester and $20 \%$ stainless steel fibres produced by the Belgian manufacturer Bekaert. This yarn exhibits a typical value of resistance of about $50 \Omega / \mathrm{cm}$. The second yarn is known as Elitex and produced by TITV, and has much lower values of electrical resistance (in the order of tens of $\Omega / \mathrm{m}$ ). It is a textured multifilament polyamide yarn with an elastane core. Its conductivity is achieved by a silver coating.

Besides conductive textile yarns, $50 \mu \mathrm{m}$ stainless steel filaments and $100 \mu \mathrm{m}$ copper wires have also been woven into fabrics.

In terms of textile structures, the tested electrodes have been produced in woven and knitted structures. When considering single face fabrics, woven structures are generally flat and thin, whereas with knitted fabrics it is possible to achieve more voluminous structures. Particularly, with the MERZ model MBS seamless knitting machine used, it is possible to produce localised variations of the fabric's structure. This possibility has been used to develop a particularly voluminous structure for the area to be used as dry electrode and thus making the electrode area stand out of the rest of the fabric.

\section{B. Force and acceleration sensors}

To increase sensitivity for skin conductivity measurement a set of flexible force sensors SEN-09376 from Sparkfun, characterized by $1-100 \mathrm{~N}$ measurement range, were embedded on the wheelchair hand support, the sensor being disposed under the E-textile skin conductivity electrodes. Additionally, the motor activity is sensed using a 3D MEMS accelerometer, ADXL335, mounted on the microcontroller platform that provides analog signals applied to analog input of the microcontroller. A set of low-pass filters are connected to the ADXL335 analog outputs Vx, Vy, Vz in order to remove the power line frequency influence.

\section{The conditioning circuits}

To obtain the ECG and SKC signals a set of signal conditioning circuits were designed and implemented. In the ECG case the conditioning circuit is expressed by an instrumentation amplifier scheme based on INA114 whose inputs are connected to the dry E-textile electrodes disposed in each hand support of wheelchair. Two filtering methods were used to improve the signal-to-noise ratio (SNR). A wide-band ECG filter was implemented as a combination of two active filters: $6^{\text {th }}$ order Butterworth band-pass filter characterized by $\mathrm{f}_{\mathrm{c} 1}=0.3 \mathrm{~Hz}$ and $\mathrm{f}_{\mathrm{c} 2}=100 \mathrm{~Hz}$ implemented with TLC2274 operational amplifier and a $3^{\text {rd }}$ order notch filter characterized by $\mathrm{f}_{\text {notch }}=50 \mathrm{~Hz}$ based on UAF42. Considering the necessity to reduce the active filter complexity, a band-pass active filter characterized by $\mathrm{fc} 1=0.3 \mathrm{~Hz}$ and $\mathrm{fc} 2=24 \mathrm{~Hz}$ was considered for the final prototype, the $50 \mathrm{~Hz}$ active notch filter being eliminated. The gains for the amplification and filtering schemes were imposed considering the voltage input range of the acquisition module.

The skin conductivity measurement can be done for one or both hands increasing the reliability of the system and also providing additional information concerning the skin conductivity difference between right and left hand. Thus, integrated E-textile sets are connected to a two channel resistance-to-voltage converter (R/V1, R/V2) followed by a low-pass filter (LPF1, LPF2). The $R / V_{1}$ and $R / V_{2}$ are implemented using a non-inverting amplification scheme including a $0.5 \mathrm{~V}$ reference voltage that is amplified by noninverting input of the amplifier which gain is imposed by the $R$ values associated with skin conductivity. The amplification scheme gain is controlled in order to provide appropriate measurement range using a CD4052 analog multiplexer controlled by microcontroller's digital lines.

\section{Microcontroller platform}

The microcontroller platform is based on the PIC24F from Microchip that presents a set of analog inputs connected to the conditioning circuits (CC) (Fig. 2).

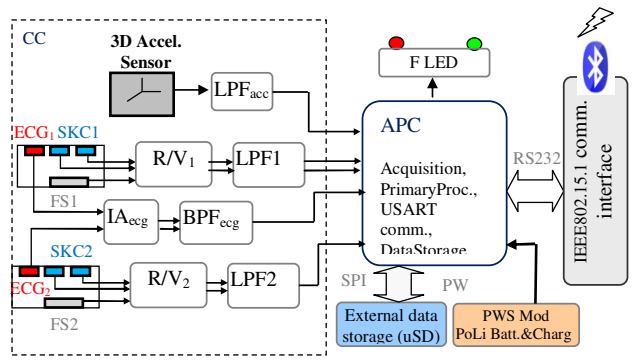

Figure 2. Block diagram of PIC24F microcontroller platform associated stress monitor embedded in the wheelchair 
In Figure 2 are presented the E-textile electrodes sets $\left(\mathrm{ECG}_{1^{-}}\right.$ $\mathrm{ECG}_{2}, \mathrm{SKC}_{1}, \mathrm{SKC}_{2}$ ), signal conditioning components of the microcontroller platform. Important tasks such as signal acquisition, primary processing, LEDs user interface control, data storage and data communication are performed by the microcontroller based on implemented firmware developed in MPLAB C30 compiler. The filtered signals are applied to AN0, AN1, AN2 e AN3 analog inputs of the microcontroller. The acquisition rate was of $200 \mathrm{~S} / \mathrm{s}$ reached through appropriate programming of TIMER 2 of the microcontroller. For accurate electrodermal response measurement the voltage signals associated with skin conductivity measurement obtained from both sensors are considered only if the user applied hand force corresponds to the baseline values.The optimal applied forces are highlighted using a set of LEDs connected to F-LED interface controlled by RF0 and RF1 digital lines. The ECG signal amplified $\left(\mathrm{IA}_{\mathrm{ecg}}\right)$ and filtered $\left(\mathrm{BPF}_{\mathrm{ecg}}\right)$ is acquired using the AN4 analog input channel of the microcontroller and processed in order to extract the information about heart rate and ECG R-R time intervals. The signals delivered by MEMS accelerometer through the $\mathrm{Vx}, \mathrm{Vy}, \mathrm{Vz}$ outputs are acquired using the AN9, AN10 and AN11 analog input using the same sampling rate that is used in the ECG acquisition rate. The implemented embedded primary processing software uses the ECG acquired samples to extract heart rate (HR) values, and the heart rate variability (HRV) through time domain spectral analysis (wavelets transform) of time intervals variation between successive peaks.

The implemented heart rate calculation algorithm includes the following steps: mean $\left(\mathrm{V} \_\right.$ecg $\left.(\mathrm{t})\right)$ and $\max \left(\mathrm{V} \_\mathrm{ecg}(\mathrm{t})\right)$ calculation for $2.5 \mathrm{~s}$ time interval; threshold $\left(\mathrm{th}_{\mathrm{a}}\right)$ calculation for specified time interval:

$$
\left.t h_{a}\right|_{\Delta t}=\frac{1}{2}\left(\text { mean }\left(V_{-} e c g(t)\right)+\max \left(V_{-} e c g(t)\right)\right.
$$

Based on calculated adaptive threshold the the stamp of ECG peak is detected, followed by time interval representation between two successive R peaks detected for $5 \mathrm{~s}$ time interval, and HR calculation.

The acquired voltage samples (V_SKC, V_ecg, V_FS, Vx, $\mathrm{Vy})$ and calculated data on the microcontroller level including the time interval between two successive ECG peaks (DELTA), and heart rate value (HR) are stored in a 10 bytes data array (Figure 3).

\begin{tabular}{|c|c|c|c|c|c|c|c|c|c|c|}
\hline $\mathbf{0}$ & $\mathbf{1}$ & $\mathbf{2}$ & $\mathbf{3}$ & $\mathbf{4}$ & $\mathbf{5}$ & $\mathbf{6}$ & $\mathbf{7}$ & $\mathbf{8}$ & $\mathbf{9}$ & $\mathbf{1 0}$ \\
\hline I & ECG & DT & HR & SKC1 & FS1 & SKC2 & FS2 & ax & ay & az \\
\hline
\end{tabular}

Figure 3. APC data array format

The I byte is used to store additional information regarding the APC functioning (e.g. battery low, applied force on the wheelchair hand support low). The ECG byte corresponds to the ECG sample, DT is the time duration between ECG peaks, $\mathrm{HR}$ is the heart rate, SKC1, SCK2 are samples of skin conductivity measured on the wheelchair hand support 1 and 2, FS1, FS2 are force samples, and ax, ay, and az are the accelerations on the $\mathrm{X}, \mathrm{Y}, \mathrm{Z}$ axis.
Using a FireFly Bluetooth module connected to the USART port of PIC24F microcontroller the data array is transmitted to the server application. The tested acquisition rate was up to 200 update/s, the data transmission rate being 19200bps.

The developed system that infers psychophysiological status from autonomic nervous system contribution on behavior and emotional predisposition is based on the Beauchaine [3] integrated model of autonomic functioning. The variables that we included in the model are: heart rate; heart rate variability (HRV); low frequency component of HRV (LF_HRV) associated with sympathetic outflow to the heart; high frequency component associated with parasympathetic outflow to the heart ( $\left.\mathrm{HF}_{-\mathrm{HRV}}\right)$; baseline skin conductance level (SCL) and event-related skin conductance response (SCR) as momentary fluctuations in skin resistance; baseline skin potential level (SPL) and event-related skin potential response (SPR) as level of skin potential at any point in time. A challenge of our future work is generalization - applying knowledge from one context to new context in order to allow engagement in biofeedback therapy and bio-cybernetic adaptation.

\section{RESULTS AND DISCUSSIONS}

The experimental work on electrical features characterization of E-textile electrodes in the wheelchair embedded system was carried out. Several tests were done with two types of ETextile conductive yarns for static and dynamic conditions (stopped wheelchair, in motion wheelchair) and also for different stress conditions.
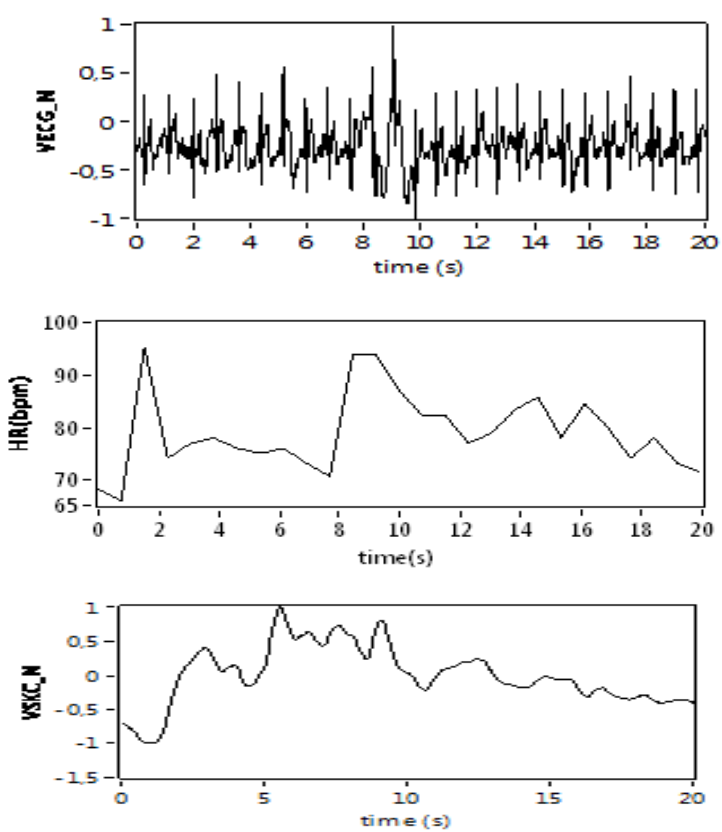

Figure 4. ECG, HR and SKC response to pleasure stimuli 
In Figure 4 slight increasing of HR and SCK is observed as response to pleasure stimuli (video clip). In addition, the variation in heart rate variability is also used together with skin conductivity to characterize affective state change. A central design for Affective Recognition and Biofeedback is represented in figure 5 .

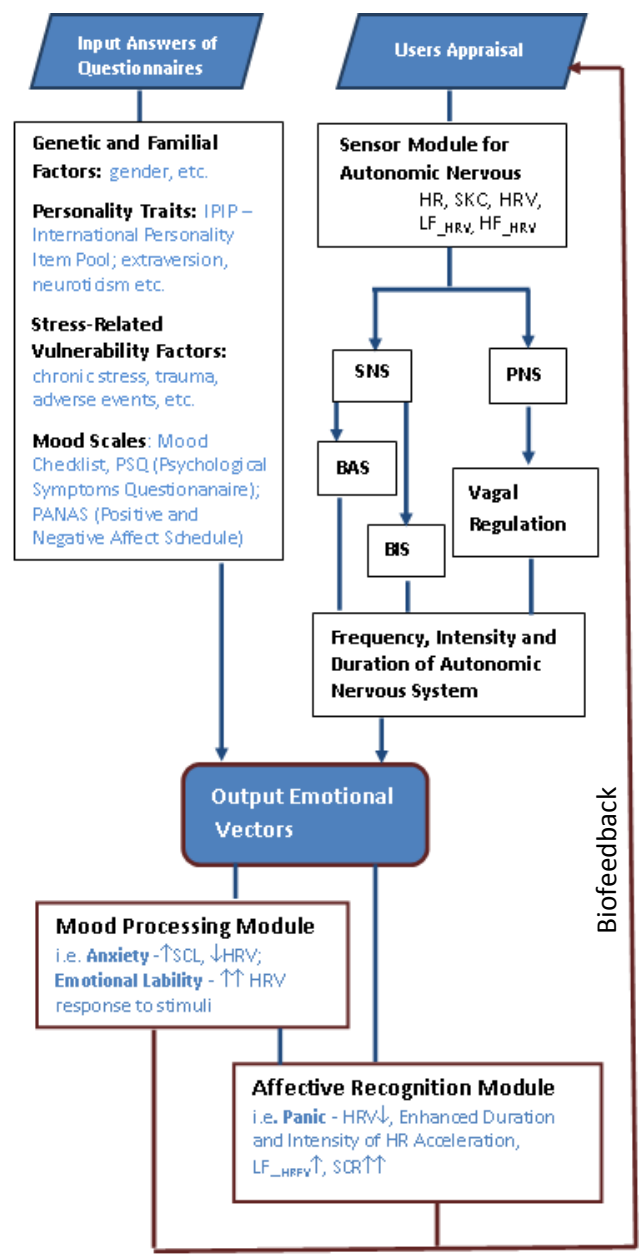

Figure 5. Affective Model Design. Physiological signal (HR- Heart Rate; SKC - Skin Conductivity, HRV - Heart Rate Variability; LF_HRV-low frequency component of HRV associated with sympathetic outflow to the heart; HF_HRV - high frequency component of HRV associated with parasympathetic branch of autonomic nervous system) are used in order to characterize Behavioral Activation System (BAS), Behavioral Inhibition System (BIS), and Vagal Regulation System. Baseline skin conductance level (SCL) are used in mood processing module, while event-related skin conductance response (SCR) is used on processing emotional response to stimuli.

The system based on Beauchaine model should detect psychophysiological stress of the user, panic, depression, aggression, extraversion events and emotional lability. The information on affective state is used as biofeedback approach for affective state visualisation and for bio-cybernatically adaptation of the system to the user affective state. For instance, in stressful situation the system provides the user with a dedicated, personal advice page (Figure 6). When this page is loaded a link to the phone number of contact person it is activated. The user can choose to deactivate this call and consult positive message included in the page or to visualize document collection (photo slideshow, video clip collection).

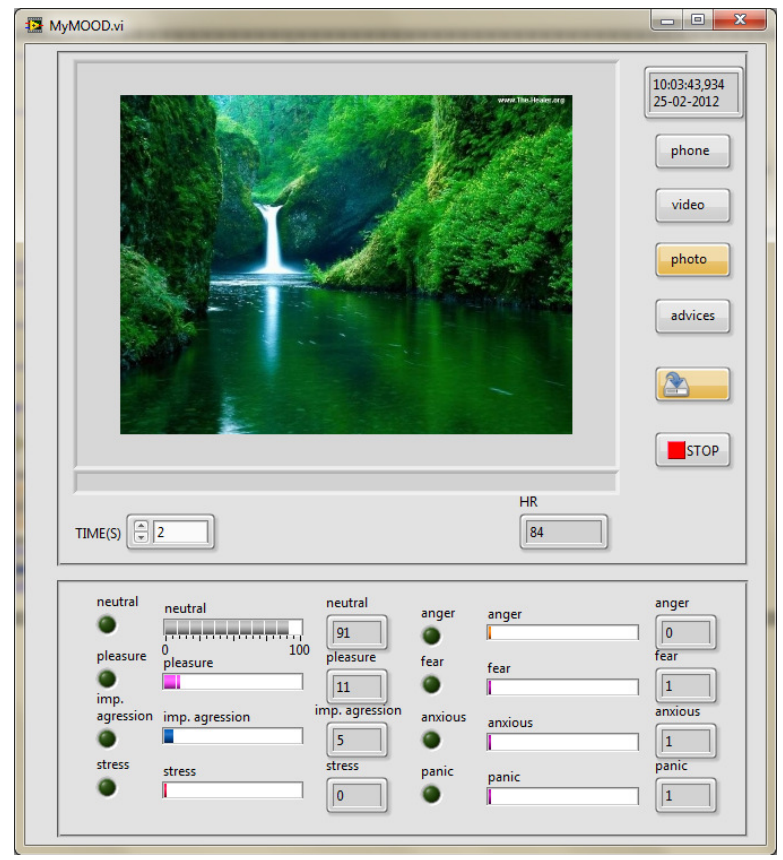

Figure 6 Example of biofeedback graphical interface developed in LabVIEW

\section{CONCLUSIONS AND FUTURE WORK}

The design and preliminary implementation of affective and physiological computing for wheelchair users using integrated E-textile electrodes as part of ECG and skin conductivity measurement system embedded in a wheelchair are presented in this paper. A microcontroller platform based on PIC24F performs acquisition of the ECG, skin conductivity, and force and acceleration signals and primary processing and Bluetooth communication. Advanced data processing including the wavelet filtering was included as part of a server application. Examples of biofeedback approach and bio-cybernetic adaptation of the system for user emotions control are described.

\section{ACKNOWLEDGEMENTS}

This work was developed under Fundação para a Ciencia e para a Tecnologia Instituto de Telecomunicações project RIPD/APD/109639/2009 and also was supported by Instituto de Telecomunicações. This support is gratefully acknowledged. 


\section{REFERENCES}

[1] K. Boehner, R. DePaula, P. Dourish, and P. Sengers, "Affect: from information to interaction", Proceedings of the $4^{\text {th }}$ Decennial Conference on Critical Computing: between Sense and Sensibility, pp.59-68, 2005.

[2] R. Picard, Affective Computing, MIT Press, 1997.

[3] T. Beauchaine, "Vagal tone, development, and Gray's motivational theory: towards an integrated model of autonomic nervous system functioning in psychopathology", Development and Psychopathology, 13, pp.183-214, 2001.

[4] S.W. Porges, "The polyvagal theory: New insights into adaptive reactions of the autonomic nervous system", Cleveland Clinic Journal of Medicine, 76(2), pp.S86-S90, 2009.

[5] S. Mahlke, M. Minge, M. Thuring, "Measuring multiple components of emotion in interactive contexts", Proceedings of CHI 2006, Canada, ACM, 2006 .

[6] G. Varni, G. Volpe, A. Camurri, "A system for real time multimodal analysis of nonverbal affective social interaction in user-centric media", IEEE Transactions on Multimedia, 12 (6), pp.576-593, 2010.

[7] R. Kaliouby, R. Picard, S. Baron-Cohen, "Affective Computing and Autism", Ann. N.Y. Acad. Sci, 1093, pp. 228-248, 2006.

[8] J. Anttonen, V. Surakka, "Emotions and heart rate while sitting on a chair", CHI2005.

[9] O. Postolache, G. Postolache, P.S.Girão, "New device for assessment of autonomic nervous system functioning in psychophysiology", Proceedings of MEMEA 2007, pp.1-4, 2007

[10] J. Allanson, S.H. Fairclough, "A research agenda for physiological computing", Interacting with Computers, 16, pp.857-878, 2004.

[11] T. Bowman, "Meet physical therapy", Communication of the ACM, 40 (8), pp.515-526, 1997.

[12] T.W. Nelson, L.J. Hettinger, J.A. Cunningham, M.M. Roe, L.G. Lu, M.W. Haas, L.B. Dennis, H.L. Pick, A. Junker, "Brain-body actuated control: assessment of an alternative control technology for virtual environments",
Image
Conference
AZ,
pp.225-232,
1996.

[13] V. Gerasimov, Every sign of life. PhD Thesis, MIT, 2003

[14] M. Catrysse, R. Puers, C. Hertleer, L. van Langenhove, H. van Egmond, D. Matthys, "Towards the integration of textile sensors in a wireless monitoring suit", Sensors and Actuators A 114, pp.302-311, 2004.

[15] M. J. Abreu, H. Carvalho, A. Catarino, A. Rocha, "Integration and embedding of vital signs sensors and other devices into textiles", Medical and Healthcare Textiles, Woodhead Publishing Textile Series Nr.75, Editors J. F. Kennedy, S. C. Anand, M. Miraftab and S. Rajendran, 2010.

[16] M. Silva, A. Catarino, H. Carvalho, A. Rocha, J. Monteiro, G. Montagna, "Textile sensors for ECG and respiratory frequency on swimsuits", Proceedings of the Conference on Intelligent Textiles and Mass Customisation, Casablanca, Morocco, 2009.

[17] P.J. Xu, H. Zhang, X.M. Tao, "Textile-structured electrodes for electrocardiogram", Textile Progress, 40(4), pp.183 -213, 2008.

[18] J., Mühlsteff, O. Such, "Dry electrodes for monitoring of vital signs in functional textiles", Proceedings of the 26th Annual International Conference of the IEEE EMBS, San Francisco, USA, 2004.

[19] G. Montagna, A. Catarino, H. Carvalho, A. Rocha, "Study and optimization of swimming performance in swimsuit designed with seamless technology", Autex 2009 World Textile Conference, Izmir, Turkey, 2009.

[20] M. Silva, A. Catarino, H. Carvalho, A. Rocha, J. Monteiro, G. Montagna, "Study of vital sign monitoring with textile sensor in swimming pool environment", Proceedings of the IECON 2009, The 35th Annual Conference of The IEEE Industrial Electronics Society, Portugal, 2009.

[21] T. Pereira, P. Silva, H. Carvalho, M. Carvalho, "Textile moisture sensor matrix for monitoring of disabled and bed-rest patients", Proceedings of the IEEE Eurocon 2011 Conference, Lisbon, Portugal, 2011. 